\title{
Pratiques d'éleveurs et résultats économiques d'élevage dans les exploitations urbaines et périurbaines de Niamey
}

\author{
A.A. MAMAN LAWAL ${ }^{1,2^{*}}$, M. CHAIBOU ${ }^{2}$, M. MANI $^{3}$, M.M. GARBA ${ }^{4}$ et \\ A.S. GOURO ${ }^{2}$ \\ ${ }^{I}$ Département des Productions Animales, Institut National de la Recherche Agronomique du Niger, \\ BP 429, Niamey, Niger. \\ ${ }^{2}$ Faculté d'Agronomie, Université Abdou Moumouni de Niamey, BP 10960, Niamey, Niger. \\ ${ }^{3}$ Faculté des Sciences Agronomiques, Université de Tahoua, BP Tahoua, Niger. \\ ${ }^{4}$ Centre de Multiplication du Bétail du Niger, BP 827, Niamey, Niger. \\ *Auteur correspondant ; E-mail : malawa80@gmail.com ; Tel : +22796992440.
}

\section{RESUME}

Cette étude a été conduite afin de caractériser les pratiques d'élevages dans la communauté urbaine de Niamey. Il a été réalisé une enquête transversale à l'issue de laquelle 344 éleveurs ont été enquêtés dans 27 quartiers et 9 villages agricoles. Les résultats ont montré que la pratique d'élevage repose le plus souvent sur la passion $(50,19 \%)$ et l'héritage $(45,65 \%)$ respectivement en milieu urbain et périurbain. L'élevage ovin prédomine et l'allotement est quasi inexistant. Les animaux sont majoritairement élevés soit dans des habitats couverts en matériaux provisoires, soit sans habitat. La main d'œuvre utilisée est principalement familiale $(86,51 \%)$ en milieu urbain et $(58,06 \%)$ en périurbain. Le stockage de l'alimentation est fortement pratiqué. L'alimentation des animaux à l'auge prédomine en milieu urbain alors qu'en milieu périurbain, la conduite alimentaire est fonction des saisons. La vaccination et le déparasitage des animaux sont fortement pratiqués. La marge brute d'un éleveur en milieu urbain s'élève à 44421,5 \pm 40236,66 FCFA par mois. Elle est de 7973,59 \pm 18890,98 FCFA pour un éleveur périurbain de Niamey. Il ressort de cette étude que l'élevage autour de la communauté urbaine de Niamey est pratiqué de façon traditionnelle avec des orientations de production pas très bien définit.

(C) 2018 International Formulae Group. All rights reserved.

Mots clés: Gestion du cheptel, conduite alimentaire, pratiques sanitaires, marge brute, éleveur, Niger.

\section{Livestock practices and economic breeding outcomes in urban and periurban farms in Niamey}

\begin{abstract}
This study was conducted to characterize livestock farming practices in the urban community of Niamey. A cross-sectional survey was conducted after which 344 breeders were surveyed in 27 districts and 9 agricultural villages. The results showed that the practice of breeding is most often based on passion $(50.19 \%)$ and inheritance $(45.65 \%)$ respectively in urban and periurban areas. Sheep farming predominates and the allotment is almost non-existent. Animals are mostly raised either in habitats covered by non-permanent
\end{abstract}


materials or without habitat. The workforce used is mainly family $(86.51 \%)$ in urban areas and $(58.06 \%)$ in the periurban area. Storage of food is highly practiced. Animal feeding at the trough is predominant in urban areas, while in periurban areas eating is seasonal. Vaccination and deworming of animals are widely practised. The gross margin of a farmer in an urban area is about $44421.5 \pm 40236.66$ FCFA per month. It is $7973.59 \pm$ 18890.98 FCFA for a periurban breeder in Niamey. This study reveals that breeding around the urban community of Niamey is practised in a traditional way with production guidelines not very well defined.

(C) 2018 International Formulae Group. All rights reserved.

Keywords: Livestock management, feeding behavior, sanitary practices, gross margin, breeder, Niger.

\section{INTRODUCTION}

Dans les pays tropicaux, l'élevage occupe une place très importante dans les activités agricoles des ménages (Zoffoun et al., 2013). Au Niger, il constitue un pôle important de l'économie nationale car occupant plus de $87 \%$ de la population et faisant vivre de façon exclusive $20 \%$ de cette population (Niger, 2013a ; Niger, 2013b). Il est une source de revenus considérables pour les populations et joue un rôle important sur le plan socioculturel. Avec une valeur de capital bétail estimée à plus de 2000 milliards de FCFA, l'élevage est le sous-secteur le plus dynamique du secteur primaire et le plus porteur de croissance pour l'économie nationale (Niger, 2007). Cependant, la persistance des cycles de sécheresse a entraîné ces dernières années, la descente des éleveurs en zone agricole et leur établissement de façon quasi permanente dans les centres urbains et périurbains. Cela a pour conséquences la sédentarisation des éleveurs mais aussi le transfert de propriété du bétail, des populations nomades et transhumantes vers les populations sédentaires (Steinfeld et al., 2010). Aussi, sous l'effet du changement climatique, il est observé une certaine variabilité dans les ressources disponibles pour le bétail (Habou et al., 2016). Il se développe alors autour de la communauté urbaine de Niamey un élevage de type urbain et périurbain à caractère semi intensif avec une forte utilisation de concentrés (Chouidi, 2011; Siddo, 2017). Bien que l'élevage urbain et périurbain représente une composante non négligeable de sécurité alimentaire et financière des populations (Waters-Bayers, 2000), cette mutation n'a pas été accompagnée d'une évolution des pratiques d'élevage des ruminants.

Ainsi, pour mieux apprécier les principales spéculations de cet élevage, il est important de connaître son organisation et ses orientations au niveau des éleveurs. La présente étude rentre dans ce cadre et a pour objectif de caractériser les pratiques d'élevages des ruminants autour de la communauté urbaine de Niamey.

\section{MATERIEL ET METHODES Zones d'étude}

L'étude a été conduite dans la communauté urbaine de Niamey (CUN) qui est composée d'une zone urbaine et d'une zone périurbaine. La zone urbaine correspond à la grande agglomération de Niamey qui est subdivisée en cinq communes (Niger, 2016). La zone périurbaine comprend tous les villages administrativement rattachés aux communes et qui sont situés entre 5 et $15 \mathrm{~km}$ autour de la ville de Niamey (Beidari, 1999). La CUN est une localité qui est située dans la partie ouest du pays, entre $2^{\circ} 10^{\prime}$ et $2^{\circ} 14^{\prime}$ de longitude Est et $13^{\circ} 33^{\prime}$ et $13^{\circ} 36^{\prime}$ de latitude Nord (Beidari, 1999 ; Niger, 2015a). Elle couvre une superficie d'environ 55227 ha avec un climat tropical du type Soudanosahélien caractérisé par une température moyenne de $30,25{ }^{\circ} \mathrm{C}$, un maximum de 42,1 ${ }^{\circ} \mathrm{C}$ en avril et un minimum de $17,8^{\circ} \mathrm{C}$ en décembre. La pluviométrie moyenne est de 563,3 mm/an (Niger, 2015a). La végétation est de façon générale arbustive, clairsemée avec des herbacées à apparition saisonnière. L'agriculture est pratiquée de manière traditionnelle et occupe une bonne frange de la population. Quant à l'élevage, il occupe une place prépondérante dans l'activité de la 
population, et le cheptel est estimé en 2015 à environ 105212 UBT (Niger, 2015b). La Figure 1 ci-dessous présente la localisation de la zone d'étude.

\section{Collecte des données}

Les données ont été collectées au moyen d'une enquête transversale conduite dans les cinq arrondissements de la CUN. Elle a concerné 27 quartiers sur les 65 et 9 villages sur les 27 que compte la communauté urbaine. La liste des quartiers et villages a été fournie par les services techniques administratifs. $\mathrm{Au}$ niveau de chaque arrondissement, $1 / 3$ des quartiers et villages ont été tirés au hasard et au niveau de chaque quartier et village, 10 éleveurs ont été enquêtés de façon aléatoire à partir d'une liste fournie par les chefs des quartiers et villages. Les informations recherchées portent essentiellement sur les caractéristiques socioéconomiques des enquêtés ; l'approvisionnement en aliment pour bétail, la conservation, le stockage et le mode d'utilisation des aliments bétail. Il a également été recherché, des informations sur le mode de gestion du troupeau et les pratiques d'élevage. Au total, 344 éleveurs ont été enquêtés dans la communauté urbaine de Niamey.

\section{Analyse statistique}

Les données collectées ont été codifiées et saisies dans une maquette réalisée sur le logiciel SPSS version 19. Une statistique descriptive (Test de Khi2; Test exact de Fisher au seuil de 5\%) et une ANOVA ont été effectué entre les variables. Les moyennes ont été comparées selon le test de Duncan au seuil de 5\%. Certaines données ont été exportées sur le tableur Excel pour la réalisation des tableaux et figures.

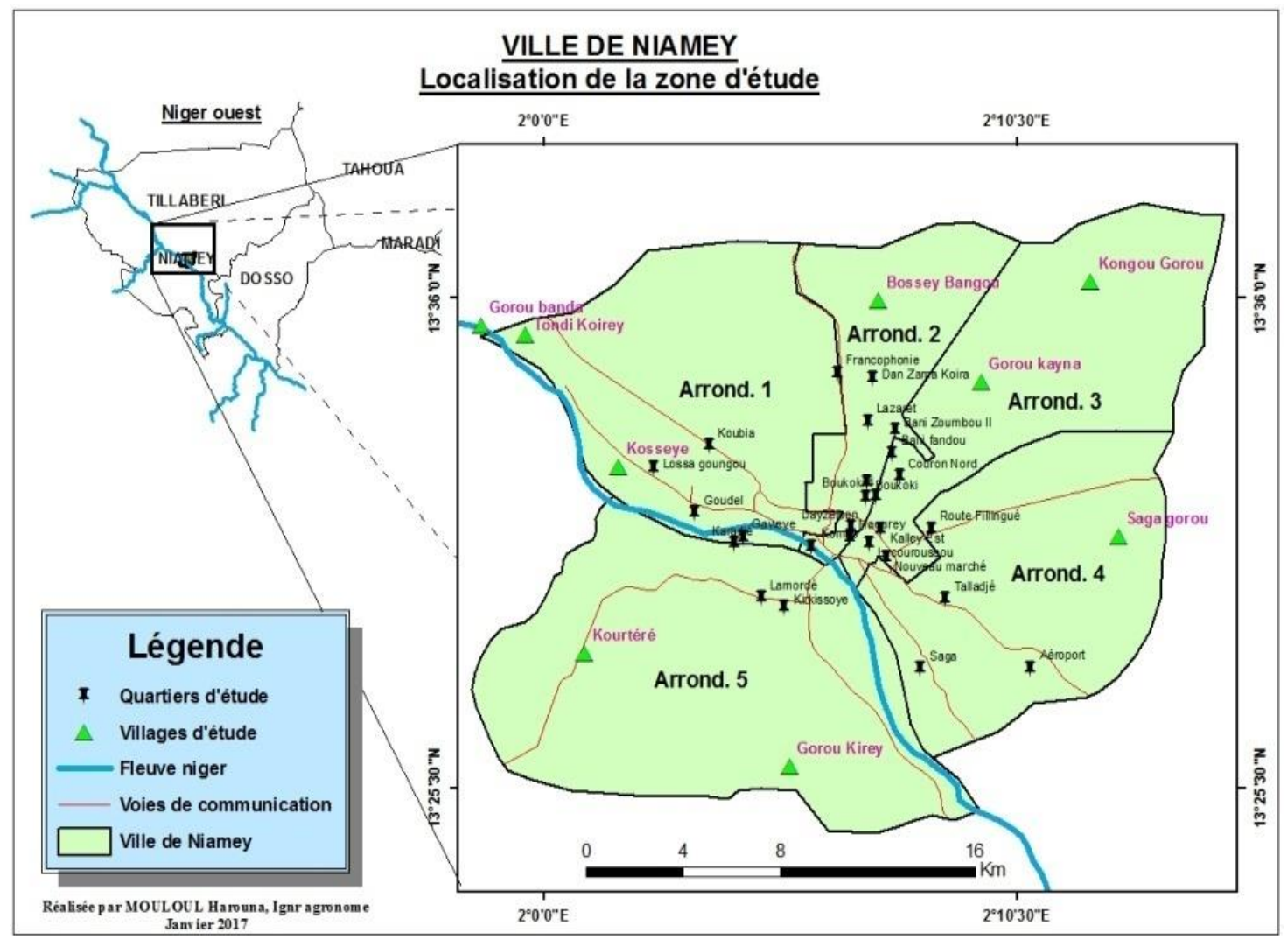

Figure 1 : Répartition des différents quartiers et village d'enquête (Lawal et al., 2017). 


\section{RESULTATS}

\section{Caractéristiques socio économiques des éleveurs}

Les éleveurs, dans leur majorité possèdent une expérience en élevage. Les Zarmas représentent l'ethnie la plus représentative $(45,6 \%)$ parmi les éleveurs urbains tandis qu'en milieu périurbain ce sont les peuls qui dominent $(69,1 \%)$. Globalement, les enquêtés étaient non instruits avec 35,7\% en milieu urbain et $43,2 \%$ en milieu périurbain (Tableau 1).

\section{Pratiques d'élevage et gestion du cheptel Objectif d'élevage}

Divers raisons conduisent à la pratique de l'élevage par la population de la communauté urbaine de Niamey. Les raisons les plus importantes sont la passion $(50,19 \%)$ en milieu urbain et l'héritage $(45,65 \%)$ en milieu périurbain. Il existe cependant une petite portion des éleveurs urbains qui pratiquent cette activité pour la fête de tabaski $(1,14 \%)$; l'épargne $(2,28 \%)$ ou pour la valorisation des résidus de cuisine $(0,76 \%)$ (Tableau 2).

\section{Appartenance des animaux}

Le cheptel appartient en grande partie aux chefs de famille $(54,8 \%$ en zone urbaine et $37 \%$ en zone périurbaine) et aux femmes ( $26,2 \%$ en milieu urbain et $39,5 \%$ en milieu périurbain). L'appartenance des animaux diffèrent selon le milieu d'élevage $(\mathrm{P}<0,001)$ (Tableau 3).

\section{Composition du cheptel d'élevage}

Il est observé, une prédominance de l'élevage des ovins avec $47,15 \%$ et $34,57 \%$ respectivement en milieu urbain et périurbain. L'élevage bovin spécifique est faible en milieu urbain $(3,42 \%)$ comme en milieu périurbain $(6,17 \%)$. Il est relativement important en élevage mixte bovins-ovins en milieu périurbain $(32,10 \%)$ (Tableau 4).

\section{Répartition des effectifs du cheptel}

Les éleveurs urbains et périurbains de Niamey possèdent plus des petits ruminants femelles. Ainsi, les moyennes des ovins

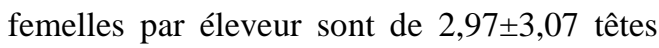
en milieu urbain et $3,60 \pm 4,58$ têtes en milieu périurbain. Celles des caprins femelles sont de

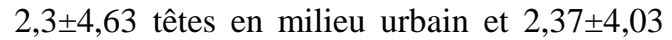
têtes en milieu périurbain. L'ANOVA a montré une différence significative entre la moyenne des bovins femelles appartenant aux éleveurs urbains $(1 \pm 3,40)$ et périurbains $(3,16 \pm 7,46)$ (Tableau 5).

\section{Agrégation des animaux}

Il s'agit, ici, du type de regroupements des animaux, des différents types de logement et de la nature de la stabulation. Les résultats montrent que plus de $80 \%$ (88,59\% en milieu urbain et $80,25 \%$ en milieu périurbain) des enquêtés élèvent leurs animaux ensemble sans distinction de sexe, d'espèce ou de catégorie. Les animaux sont élevés en grande partie dans des habitats couverts en matériaux provisoires $(57,03 \%$ en zone urbaine et $25,93 \%$ en zone périurbaine), ou à l'air libre sans habitat $(18,63 \%$ en zone urbaine et $46,91 \%$ en zone périurbaine), $(\mathrm{P}<0,001)$. La stabulation est entravée $(49,05 \%$ en milieu urbain et $49,38 \%$ en milieu périurbain) ou à la fois libre et entravée $(27,38 \%$ en milieu urbain et $27,16 \%$ en milieu périurbain) (Tableau 6).

\section{Gestion de la main d'auvre}

Dans la zone urbaine de Niamey, $47,91 \%$ des enquêtés utilisent la main d'œuvre dans la conduite de l'élevage, contre 38,27\% en zone périurbaine. La main d'œuvre est majoritairement utilisée pendant toutes les saisons $(94,44 \%$ en milieu urbain et $48,39 \%$ en milieu périurbain). Néanmoins, en milieu périurbain, on observe une importante proportion d'utilisateurs de cette main d'œuvre $(35,48 \%)$ uniquement pendant la saison sèche $(\mathrm{P}<0,001)$. Cependant, elle est principalement familiale avec $86,51 \%$ des cas en milieu urbain et $58,06 \%$ en milieu périurbain $(\mathrm{P}<0,001)$ (Tableau 7).

\section{Pratiques alimentaires Gestion du stock alimentaire}

Il ressort des résultats de cette étude, que plus de $70 \%$ des enquêtés $(73,76 \%$ des éleveurs urbains et $77,78 \%$ des éleveurs périurbains) pratiquent le stockage de l'alimentation pour les animaux. A cet effet, plusieurs catégories d'aliments dans des combinaisons différentes font l'objet de stockage. Ainsi, la combinaison résidus de 
culture (RC) et Sous produits agroindustriels (SPAI) est la plus pratiquée en milieu urbain $(37,11 \%)$ comme en milieu périurbain $(42,2 \%)$. Cette proportion est suivie par le stockage des résidus de culture simple en milieu périurbain avec $32,8 \%$, et en milieu urbain par celle combinant les résidus de culture, les sous produits agroindustriels et la paille de brousse (PB), ( $\mathrm{P}<0,001)$. Les pratiques du stockage d'aliment pour bétail sont présentées dans le Tableau 8.

\section{Traitement du fourrage}

Dans certaines situations, le fourrage est traité avant d'être distribué aux animaux. A travers cette étude, il ressort que le traitement de fourrage est faiblement pratiqué par les enquêtés $(9,27 \%$ et $25,92 \%$, respectivement en milieu urbain et périurbain). Cette situation est significative au seuil de $5 \%$ ( $\mathrm{P}<0,01)$. Le salage $(47,83 \%$ en milieu urbain et $61,90 \%$ en milieu périurbain) suivit du tri $(26,09 \%$ en milieu urbain et $19,05 \%$ en milieu périurbain) sont les traitements les plus pratiqués par les éleveurs (Tableau 9).

\section{Conduite alimentaire}

Les éleveurs en milieu urbain, à plus de $70 \%$ alimentent leurs animaux à l'auge pendant toute l'année, mais c'est en milieu périurbain qu'il ya une certaine variabilité de mode de conduite en fonction des saisons. En saison des pluies, les animaux sont plus alimentés à l'auge $(47,83 \%)$ ou conduits au parcourt naturel par un berger $(40,22 \%)$, $(\mathrm{P}<0,001)$. En saison sèche, on observe une baisse de la pratique d'alimentation à l'auge et une augmentation de la divagation des animaux $(\mathrm{P}<0,001)$ (Tableau 10).

\section{Complémentation/supplémentation}

Les résultats de l'étude révèlent que le concentré le plus utilisé en alimentation animale dans la communauté urbaine de Niamey est le son de céréale. Il est majoritairement distribué aux animaux deux fois par jour $(42,6 \%$ en zone urbaine et $65,4 \%$ en zone périurbaine) sans distinction de période $(92,4 \%$ en zone urbaine et $76,5 \%$ en zone périurbaine). Les tourteaux (d'arachide et de coton) et les cosses de niébé sont faiblement utilisés. Quand aux fourrages, en milieu urbain, ce sont les fanes de niébé et la paille de brousses qui sont abondement utilisées sans préférence de saison. La complémentation avec les fourrages en milieu périurbain de Niamey est relativement faible, de même que la supplémentation en éléments minéraux dans la communauté urbaine de Niamey (Tableau 11).

\section{Pratiques sanitaires Vaccination}

Les résultats ont montré que la majeure partie des éleveurs de la communauté urbaine de Niamey $(75,70 \%$ des éleveurs urbains et 91,36\% des éleveurs périurbains) vaccinent leurs animaux et le plus souvent pendant la campagne gratuite réalisée par l'Etat $(45,73 \%$ en milieu urbain et $64,86 \%$ en milieu périurbain). Une proportion de $25,13 \%$ des éleveurs urbains qui pratiquent la vaccination le font avant la saison pluvieuse (Tableau 12).

\section{Déparasitage}

Le déparasitage est pratiqué par $75,67 \%$ et $91,40 \%$ des éleveurs enquêtés, respectivement en milieu urbain et périurbain de Niamey. En zone urbaine, 36,70\% le font avant la saison pluvieuse et $25,10 \%$ en fonction de l'état des animaux. En milieu périurbain, c'est plutôt pendant la campagne gratuite de l'Etat $(35,14 \%)$ et avant la saison pluvieuse $(31,08 \%)$ que le déparasitage est effectué (Tableau 13).

\section{Déterminants de la marge brute par éleveur}

Les principales charges liées à l'alimentation des animaux dans la communauté urbaine de Niamey sont, entre autres, les charges liées à la main d'œuvre, la conduite au pâturage, l'achat et le transport de l'alimentation; mais aussi les soins administrés aux animaux. Le chiffre d'affaire, quant à lui, repose essentiellement sur la vente des animaux sur pieds, celle du lait et du fumier. Ainsi, la marge brute d'un éleveur en milieu urbain s'élève à 44421,5 40236,66

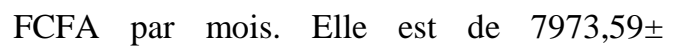
18890,98 FCFA pour un éleveur périurbain de Niamey (Tableau 14). 
Tableau 1 : Caractéristiques socio-économiques des enquêtés.

\begin{tabular}{|c|c|c|c|c|c|c|}
\hline \multirow{2}{*}{ Variables } & \multirow{2}{*}{ Modalités } & \multicolumn{2}{|c|}{ Urbain } & \multicolumn{2}{|c|}{ Périurbain } & \multirow{2}{*}{$\mathbf{P}$} \\
\hline & & $\mathbf{N}$ & $\%$ & $\mathbf{N}$ & $\%$ & \\
\hline \multirow{3}{*}{ Sexe } & Masculin & 156 & 59,3 & 34 & 42 & \multirow{3}{*}{$* *$} \\
\hline & Féminin & 107 & 40,7 & 47 & 58 & \\
\hline & Total & 263 & 100 & 81 & 100 & \\
\hline \multirow{7}{*}{ Ethnie } & Haoussa & 79 & 30 & 2 & 2,5 & \multirow{7}{*}{$* * *$} \\
\hline & Zarma & 120 & 45,6 & 20 & 24,7 & \\
\hline & Peul & 51 & 19,4 & 56 & 69,1 & \\
\hline & Touareg & 9 & 3,4 & 3 & 3,7 & \\
\hline & Gourmantché & 1 & 0,4 & - & - & \\
\hline & Kanouri & 3 & 1,1 & - & - & \\
\hline & Total & 263 & 100 & 81 & 100 & \\
\hline \multirow{6}{*}{ Niveau de d'instruction } & Aucun & 94 & 35,7 & 35 & 43,2 & \multirow{6}{*}{ Ns } \\
\hline & $\begin{array}{l}\text { Alphabétisé arabe et/ou } \\
\text { traditionnel }\end{array}$ & 69 & 26,2 & 20 & 24,7 & \\
\hline & Primaire & 48 & 18,3 & 19 & 23,5 & \\
\hline & Secondaire & 38 & 14,4 & 4 & 4,9 & \\
\hline & Supérieur & 14 & 5,3 & 3 & 3,7 & \\
\hline & Total & 263 & 100 & 81 & 100 & \\
\hline \multirow{5}{*}{ Niveau de décision } & Chef de famille & 114 & 43,3 & 23 & 28,4 & \multirow{5}{*}{$* * *$} \\
\hline & Chef de ménage indépendant & 74 & 28,1 & 23 & 28,4 & \\
\hline & Chef de ménage dépendant & 57 & 21,7 & 32 & 39,5 & \\
\hline & Actif du ménage & 18 & 6,8 & 3 & 3,7 & \\
\hline & Total & 263 & 100 & 81 & 100 & \\
\hline
\end{tabular}

Tableau 2 : Les raisons de la pratique d'élevage dans la communauté urbaine de Niamey.

\begin{tabular}{llccccc}
\hline \multirow{2}{*}{ Variables } & \multirow{2}{*}{ Modalités } & \multicolumn{2}{c}{ Urbain } & \multicolumn{2}{c}{ Périurbain } & \multirow{2}{*}{ P } \\
\cline { 2 - 5 } & & $\mathbf{N}$ & $\mathbf{\%}$ & $\mathbf{N}$ & $\mathbf{\%}$ & \\
\hline & Tradition & 71 & 27 & 37 & 45,68 & \\
& Passion & 132 & 50,19 & 21 & 25,93 & \\
& Contrainte & 13 & 4,94 & 6 & 7,41 & \\
Raisons de la & Génère revenu & 36 & 13,69 & 17 & 20,99 & \\
pratique d'élevage & Epargne & 6 & 2,28 & - & - & $*$ \\
& Valorisation des & 2 & 0,76 & - & - & \\
& résidus de cuisine & 2 & & & & \\
& Tabaski & 3 & 1,14 & - & - & \\
& Total & 263 & 100 & 81 & 100 & \\
\hline
\end{tabular}

\footnotetext{
$* *=\mathrm{p}<0,01 ; \mathrm{N}=$ effectif $; \%=$ pourcentage.
} 
Tableau 3 : Appartenance des animaux.

\begin{tabular}{|c|c|c|c|c|c|c|}
\hline \multirow{2}{*}{ Variables } & \multirow{2}{*}{ Modalités } & \multicolumn{2}{|c|}{ Urbain } & \multicolumn{2}{|c|}{ Périurbain } & \multirow{2}{*}{$\mathbf{P}$} \\
\hline & & $\mathbf{N}$ & $\%$ & $\mathbf{N}$ & $\%$ & \\
\hline \multirow{8}{*}{ Appartenance des animaux } & Au chef & 144 & 54,8 & 30 & 37 & \multirow{8}{*}{$* * *$} \\
\hline & Aux hommes & 15 & 5,7 & - & - & \\
\hline & Aux femmes & 69 & 26,2 & 32 & 39,5 & \\
\hline & Aux enfants & 8 & 3,0 & 4 & 4,9 & \\
\hline & Aux hommes et femmes & 7 & 2,7 & 8 & 9,9 & \\
\hline & Aux femmes et enfants & 10 & 3,8 & - & - & \\
\hline & Familiale & 10 & 3,8 & 7 & 8,6 & \\
\hline & Total & 263 & 100 & 81 & 100 & \\
\hline
\end{tabular}

$* * *=\mathrm{p}<0,001 ; \mathrm{N}=$ effectif $; \%=$ pourcentage.

Tableau 4 : Type d'animaux élevés.

\begin{tabular}{llccccc}
\hline \multirow{2}{*}{ Variables } & \multirow{2}{*}{ Modalité } & \multicolumn{2}{c}{ Urbain } & \multicolumn{2}{c}{ Périurbain } & \multirow{2}{*}{$\mathbf{P}$} \\
\cline { 2 - 6 } & & $\mathbf{N}$ & $\mathbf{\%}$ & $\mathbf{N}$ & $\mathbf{\%}$ & \\
\hline \multirow{4}{*}{ Espèce animale élevée } & Bovins & 9 & 3,42 & 5 & 6,17 & \\
& Ovins & 124 & 47,15 & 28 & 34,57 & \\
& Caprins & 13 & 4,94 & 1 & 1,23 & \\
& Bovins et ovins & 22 & 8,37 & 6 & 7,41 & $* * *$ \\
& Bovins et caprins & 5 & 1,90 & - & - & \\
& Ovins et caprins & 66 & 25,10 & 15 & 18,52 & \\
& Bovins, ovins et caprins & 24 & 9,13 & 26 & 32,10 & \\
& Total & 263 & 100 & 81 & 100 & \\
\hline
\end{tabular}

$* * *=\mathrm{p}<0,001 ; \mathrm{N}=$ effectif $; \%=$ pourcentage.

Tableau 5 : Répartition du cheptel par sexe et par éleveur.

\begin{tabular}{lccc}
\hline \multirow{2}{*}{ Variables } & \multicolumn{2}{c}{ Moyenne } & \multirow{2}{*}{ P } \\
\cline { 2 - 3 } & Urbain & Périurbain & \\
\hline Le nombre de bovins mâles & $0,52 \pm 1,58$ & $0,64 \pm 1,08$ & Ns \\
Le nombre de bovins femelles & $1 \pm 3,40$ & $3,16 \pm 7,46$ & $* * *$ \\
Le nombre d'ovins mâles & $2,23 \pm 2,22$ & $2,01 \pm 1,63$ & Ns \\
Le nombre d'ovins femelles & $2,97 \pm 3,07$ & $3,60 \pm 4,58$ & Ns \\
Le nombre de caprins mâles & $0,75 \pm 1,31$ & $0,90 \pm 1,36$ & Ns \\
Le nombre de caprins femelles & $2,3 \pm 4,63$ & $2,37 \pm 4,03$ & Ns \\
\hline$* * *=p<0,001$ & & &
\end{tabular}


Tableau 6 : Agrégation des animaux.

\begin{tabular}{|c|c|c|c|c|c|c|}
\hline \multirow{2}{*}{ Variables } & \multirow{2}{*}{ Modalité } & \multicolumn{2}{|c|}{ Urbain } & \multicolumn{2}{|c|}{ Périurbain } & \multirow{2}{*}{$\mathbf{P}$} \\
\hline & & $\mathbf{N}$ & $\%$ & $\mathbf{N}$ & $\%$ & \\
\hline \multirow{5}{*}{$\begin{array}{l}\text { Type de } \\
\text { regroupement }\end{array}$} & Tous ensembles & 233 & 88,59 & 65 & 80,25 & \multirow{5}{*}{ Ns } \\
\hline & Par espèce & 27 & 10,27 & 16 & 19,75 & \\
\hline & Par catégorie & 1 & 0,38 & - & - & \\
\hline & Par espèce et catégorie & 2 & 0,76 & - & - & \\
\hline & Total & 263 & 100 & 81 & 100 & \\
\hline \multirow{7}{*}{$\begin{array}{l}\text { Types de } \\
\text { logement }\end{array}$} & $\begin{array}{l}\text { Habitat couvert en matériaux } \\
\text { définitifs }\end{array}$ & 11 & 4,18 & - & - & \multirow{7}{*}{$* * *$} \\
\hline & $\begin{array}{l}\text { Habitat couvert en matériaux } \\
\text { non définitifs }\end{array}$ & 150 & 57,03 & 21 & 25,93 & \\
\hline & Enclos à épineux & 9 & 3,42 & 6 & 7,41 & \\
\hline & Enclos en grillage & 32 & 12,17 & 14 & 17,28 & \\
\hline & pas d'habitat & 49 & 18,63 & 38 & 46,91 & \\
\hline & $\begin{array}{l}\text { Enclos en matériaux } \\
\text { définitifs }\end{array}$ & 12 & 4,56 & 2 & 2,47 & \\
\hline & Total & 263 & 100 & 81 & 100 & \\
\hline \multirow{4}{*}{$\begin{array}{l}\text { Types de } \\
\text { stabulation }\end{array}$} & Libre & 62 & 23,57 & 19 & 23,46 & \multirow{4}{*}{ Ns } \\
\hline & Entravée & 129 & 49,05 & 40 & 49,38 & \\
\hline & Libre et entravée & 72 & 27,38 & 22 & 27,16 & \\
\hline & Total & 263 & 100 & 81 & 100 & \\
\hline
\end{tabular}

Ns = non significatif $; * * *=p<0,001 ; \mathrm{N}=$ effectif $; \%=$ pourcentage.

Tableau 7 : Gestion de la main d'œuvre.

\begin{tabular}{|c|c|c|c|c|c|c|}
\hline \multirow{2}{*}{ Variables } & \multirow{2}{*}{ Modalité } & \multicolumn{2}{|c|}{ Urbain } & \multicolumn{2}{|c|}{ Périurbain } & \multirow{2}{*}{$\mathbf{P}$} \\
\hline & & $\mathbf{N}$ & $\%$ & $\mathbf{N}$ & $\%$ & \\
\hline \multirow{3}{*}{$\begin{array}{l}\text { Utilisation de } \\
\text { la main } \\
\text { d'œuvre }\end{array}$} & Oui & 126 & 47,91 & 31 & 38,27 & \multirow{3}{*}{ Ns } \\
\hline & Non & 137 & 52,09 & 50 & 61,73 & \\
\hline & Total & 263 & 100 & 81 & 100 & \\
\hline \multirow{6}{*}{$\begin{array}{l}\text { Période } \\
\text { d'utilisation de } \\
\text { la main } \\
\text { d'œuvre }\end{array}$} & Saison des pluies & 1 & 0,79 & 3 & 9,68 & \multirow{6}{*}{$* *$} \\
\hline & Saison sèche froide & - & - & 1 & 3,23 & \\
\hline & $\begin{array}{l}\text { Saison sèche } \\
\text { chaude }\end{array}$ & - & - & 1 & 3,23 & \\
\hline & Saison sèche & 6 & 4,76 & 11 & 35,48 & \\
\hline & A tout moment & 119 & 94,44 & 15 & 48,39 & \\
\hline & Total & 126 & 100 & 31 & 100 & \\
\hline \multirow{4}{*}{$\begin{array}{l}\text { Genre de la } \\
\text { main d'œuvre }\end{array}$} & Familiale & 109 & 86,51 & 18 & 58,06 & \multirow{4}{*}{$* * *$} \\
\hline & Salariale & 16 & 12,70 & 10 & 32,26 & \\
\hline & $\begin{array}{l}\text { Familiale et } \\
\text { salariale }\end{array}$ & 1 & 0,79 & 3 & 9,68 & \\
\hline & Total & 126 & 100 & 31 & 100 & \\
\hline
\end{tabular}

Ns = non significatif $; * * *=\mathrm{p}<0,001 ; \mathrm{N}=$ effectif $; \%=$ pourcentage. 
Tableau 8: Pratiques du stockage d'aliment pour bétail.

\begin{tabular}{llccccc}
\hline \multirow{2}{*}{ Variables } & Modalité & \multicolumn{2}{c}{ Urbain } & \multicolumn{2}{c}{ Village } & \multirow{2}{*}{ P } \\
\cline { 2 - 6 } & & $\mathbf{N}$ & $\mathbf{\%}$ & $\mathbf{N}$ & $\mathbf{\%}$ & \\
\hline Stockage de & Oui & 194 & 73,76 & 63 & 77,78 & \\
l'alimentation pour & Non & 69 & 26,24 & 18 & 22,22 & Ns \\
animaux & Total & 263 & 100 & 81 & 100 & \\
\hline & RC & 19 & 9,79 & 21 & 32,8 & \\
& SPAI & 8 & 4,12 & 2 & 3,1 & \\
Catégories d'aliment & RC et SPAI & 72 & 37,11 & 27 & 42,2 & $* * *$ \\
stocké & PB & 3 & 1,55 & - & - & $*$ \\
& RC et PB & 8 & 4,12 & - & - & \\
& SPAI et PB & 31 & 15,98 & 8 & 12,5 & \\
& RC; SPAI et PB & 53 & 27,32 & 6 & 9,4 & \\
\hline & Total & 194 & 100 & 64 & 100 & \\
\hline
\end{tabular}

$\mathrm{Ns}=$ non significatif $; * * *=\mathrm{p}<0,001 ; \mathrm{N}=$ effectif $; \%=$ pourcentage

Tableau 9 : Pratiques de traitement de fourrages.

\begin{tabular}{llccccc}
\hline \multirow{2}{*}{ Variables } & \multirow{2}{*}{ Modalité } & Urbain & \multicolumn{3}{c}{ Périurbain } & \multirow{2}{*}{ P } \\
\cline { 2 - 6 } & & $\mathbf{N}$ & $\mathbf{\%}$ & $\mathbf{N}$ & $\mathbf{\%}$ & \\
\hline pratique de & Oui & 23 & 9,27 & 21 & 25,92 & \\
traitement de & Non & 225 & 90,73 & 60 & 74,10 & $* *$ \\
fourrage & Total & 248 & 100,00 & 81 & 100,00 & \\
\hline & Traitement à l'urée & 2 & 8,70 & 1 & 4,76 & \\
& Salage & 11 & 47,83 & 13 & 61,90 & \\
Type de & Hachage & 2 & 8,70 & 2 & 9,52 & \\
traitement & Broyage & 2 & 8,70 & - & - & Ns \\
& Traitement à l'urée et salage & 1 & 4,76 & - & - & \\
& Tri & 6 & 26,09 & 4 & 19,05 & \\
& Total & 23 & 100,00 & 21 & 100,00 & \\
\hline
\end{tabular}

Ns $=$ non significatif $; * *=\mathrm{p}<0,01 ; \mathrm{N}=$ effectif $; \%=$ pourcentage.

Tableau 10 : Conduites alimentaires en fonction des saisons.

\begin{tabular}{|c|c|c|c|c|c|c|}
\hline \multirow{2}{*}{ Variables } & \multirow{2}{*}{ Modalité } & \multirow{2}{*}{$\begin{array}{c}\text { Urbain } \\
\mathbf{N} \\
\end{array}$} & \multicolumn{3}{|c|}{ Périurbain } & \multirow{2}{*}{$\mathbf{P}$} \\
\hline & & & $\%$ & $\mathbf{N}$ & $\%$ & \\
\hline \multirow{8}{*}{$\begin{array}{l}\text { Conduite } \\
\text { alimentaire } \\
\text { en saison } \\
\text { sèche }\end{array}$} & A l'auge & 180 & 68,44 & 20 & 24,69 & \multirow{8}{*}{$* * *$} \\
\hline & Divagation & 27 & 10,27 & 25 & 30,86 & \\
\hline & Sur parcourt naturel par un berger & 27 & 10,27 & 26 & 32,10 & \\
\hline & A l'auge et divagation & 12 & 4,56 & 1 & 1,23 & \\
\hline & A l'auge et parcourt naturel & 16 & 6,08 & 7 & 8,64 & \\
\hline & A l'auge, divagation et parcourt naturel & - & - & 1 & 1,23 & \\
\hline & Divagation et conduite & 1 & 0,38 & 1 & 1,23 & \\
\hline & Total & 263 & 100,00 & 81 & 100,00 & \\
\hline \multirow{8}{*}{$\begin{array}{l}\text { Conduite } \\
\text { alimentaire } \\
\text { en saison } \\
\text { pluie }\end{array}$} & A l'auge & 179 & 68,06 & 34 & 41,98 & \multirow{8}{*}{$* * *$} \\
\hline & Divagation & 31 & 11,79 & 9 & 11,11 & \\
\hline & Sur parcourt naturel par un berger & 26 & 9,89 & 27 & 33,33 & \\
\hline & A l'auge et divagation & 12 & 4,56 & - & - & \\
\hline & A l'auge et parcourt naturel & 13 & 4,94 & 9 & 11,11 & \\
\hline & A l'auge, divagation et parcourt naturel & 1 & 0,38 & 1 & 1,23 & \\
\hline & Divagation et conduite & 1 & 0,38 & 1 & 1,23 & \\
\hline & Total & 263 & 100,00 & 81 & 100,00 & \\
\hline
\end{tabular}


Tableau 11 : Fréquences de distribution et période d'utilisation des aliments bétail.

\begin{tabular}{|c|c|c|c|c|c|c|c|c|c|c|c|c|c|c|}
\hline \multirow{3}{*}{$\begin{array}{l}\text { Milieux } \\
\text { d'élevage }\end{array}$} & \multirow{3}{*}{$\begin{array}{l}\text { Catégories } \\
\text { d'aliment }\end{array}$} & \multirow{3}{*}{$\begin{array}{l}\text { Types } \\
\text { d'aliments }\end{array}$} & \multicolumn{6}{|c|}{ Fréquences de distribution } & \multicolumn{6}{|c|}{ Période d'utilisation } \\
\hline & & & \multicolumn{2}{|c|}{1 fois/j } & \multicolumn{2}{|c|}{2 fois/j } & \multicolumn{2}{|c|}{ NC } & \multicolumn{2}{|c|}{ SS } & \multicolumn{2}{|c|}{ SP } & \multicolumn{2}{|c|}{ SS et SP } \\
\hline & & & $\mathbf{N}$ & $\%$ & $\mathbf{N}$ & $\%$ & $\mathbf{N}$ & $\%$ & $\mathbf{N}$ & $\%$ & $\mathbf{N}$ & $\%$ & $\mathbf{N}$ & $\%$ \\
\hline \multirow{14}{*}{ Urbain } & \multirow{4}{*}{ Concentré } & TA & - & - & 4 & 1,5 & - & - & 2 & 0,8 & - & - & 2 & 0,8 \\
\hline & & TGC & 2 & 0,8 & 1 & 0,4 & - & - & - & - & - & - & 3 & 1,1 \\
\hline & & $\mathrm{SC}$ & 77 & 29,3 & 112 & 42,6 & 66 & 25,1 & 9 & 3,4 & 2 & 0,8 & 243 & 92,4 \\
\hline & & $\mathrm{CN}$ & 13 & 4,9 & 19 & 7,2 & 12 & 4,6 & 5 & 1,9 & - & - & 36 & 13,7 \\
\hline & \multirow{6}{*}{ Fourrage } & PB & 33 & 12,5 & 44 & 16,7 & 43 & 16,3 & 30 & 11,4 & 4 & 1,5 & 83 & 31,6 \\
\hline & & $\mathrm{TC}$ & 6 & 2,3 & 8 & 3 & 2 & 0,8 & 10 & 3,8 & - & - & 16 & 6,1 \\
\hline & & PR & 6 & 2,3 & 5 & 1,9 & 13 & 4,9 & 5 & 1,9 & 3 & 1,1 & 13 & 4,9 \\
\hline & & FA & 12 & 4,6 & 28 & 10,6 & 20 & 7,6 & 13 & 4,9 & - & - & 43 & 16,3 \\
\hline & & $\mathrm{FN}$ & 27 & 10,3 & 57 & 21,7 & 41 & 15,6 & 46 & 17,5 & 3 & 1,1 & 74 & 28,1 \\
\hline & & $\mathrm{HF}$ & 7 & 2,7 & 8 & 3 & 32 & 12,2 & 25 & 9,5 & 1 & 0,4 & 21 & 8 \\
\hline & \multirow{4}{*}{ Supplément } & Scu & 14 & 5,3 & 2 & 0,8 & 19 & 7,2 & 1 & 0,4 & 1 & 0,4 & 33 & 12,5 \\
\hline & & SB & 6 & 2,3 & 4 & 1,5 & 11 & 4,2 & - & - & - & - & 21 & 8 \\
\hline & & NA & 1 & 0,4 & 4 & 1,5 & 1 & 0,4 & - & - & - & - & 5 & 1,9 \\
\hline & & $\mathrm{BI}$ & - & - & 1 & 0,4 & 10 & 3,8 & - & - & 2 & 0,8 & 9 & 3,4 \\
\hline \multirow{13}{*}{ Périurbain } & \multirow{4}{*}{ Concentré } & TA & - & - & - & - & - & - & - & - & - & - & - & - \\
\hline & & TGC & 1 & 1,2 & 3 & 3,7 & - & - & 2 & 2,5 & - & - & 2 & 2,5 \\
\hline & & $\mathrm{SC}$ & 13 & 16 & 53 & 65,4 & 12 & 14,8 & 15 & 18,5 & - & - & 62 & 76,5 \\
\hline & & $\mathrm{CN}$ & 8 & 9,9 & 3 & 3,7 & - & - & 3 & 3,7 & 1 & 1,2 & 5 & 6,2 \\
\hline & \multirow{6}{*}{ Fourrage } & PB & 1 & 1,2 & 2 & 2,5 & 12 & 14,8 & 3 & 3,7 & 10 & 12 & 1 & 1,2 \\
\hline & & $\mathrm{TC}$ & 7 & 8,6 & 9 & 11,1 & 2 & 2,5 & 18 & 22,2 & - & - & - & - \\
\hline & & PR & 10 & 12,3 & 10 & 12,3 & 9 & 11,1 & 18 & 22,2 & - & - & 11 & 13,6 \\
\hline & & FA & 5 & 6,2 & 7 & 8,6 & 2 & 2,5 & 4 & 4,9 & - & - & 7 & 8,6 \\
\hline & & $\mathrm{FN}$ & 10 & 12,3 & 17 & 21 & 11 & 13,6 & 26 & 32,1 & - & - & 12 & 14,8 \\
\hline & & $\mathrm{HF}$ & - & - & 3 & 3,7 & 10 & 12,3 & - & - & 13 & 16 & - & - \\
\hline & \multirow{3}{*}{ Supplément } & Scu & 8 & 9,9 & 1 & 1,2 & 11 & 13,6 & - & - & 2 & 2,5 & 18 & 22,2 \\
\hline & & SB & 4 & 4,9 & 1 & 1,2 & 2 & 2,5 & 2 & 2,5 & - & - & 5 & 6,2 \\
\hline & & $\mathrm{BI}$ & 1 & 1,2 & - & - & 7 & 8,6 & - & - & 1 & 1,2 & 6 & 7,4 \\
\hline
\end{tabular}

$\mathrm{TA}=$ tourteau d'arachide $; \mathrm{TGC}=$ Tourteau/grain de coton $; \mathrm{SC}=$ Son de céréales $; \mathrm{CN}=$ Cosse de niébé $; \mathrm{PB}=\mathrm{Paille}$ de brousse $; \mathrm{TC}=$ Tiges des céréales $; \mathrm{PR}=$ Paille de riz $; \mathrm{FA}=$ Fanes d'arachide $; \mathrm{FN}=$ Fanes de niébé $; \mathrm{HF}=$ herbe fraiche $; \mathrm{Scu}=$ Sel de cuisine ; $\mathrm{SB}=\mathrm{Sel}$ de Bilma ; NA= Natron ; BI= Bloc industriel ; N= effectif ; \%= Pourcentage.

Tableau 12 : Pratique de vaccination des animaux.

\begin{tabular}{llccccc}
\hline \multirow{2}{*}{ Variables } & \multirow{2}{*}{ Modalité } & \multicolumn{2}{c}{ Urbain } & \multicolumn{2}{c}{ Périurbain } & \multirow{2}{*}{ P } \\
\cline { 3 - 6 } & & $\mathbf{N}$ & $\mathbf{\%}$ & $\mathbf{N}$ & $\mathbf{\%}$ & \\
\hline \multirow{2}{*}{$\begin{array}{l}\text { Vaccination } \\
\text { des animaux }\end{array}$} & Oui & 199 & 75,70 & 74 & 91,36 & \\
& Non & 64 & 24,30 & 7 & 8,64 & $*$ \\
& Total & 263 & 100 & 81 & 100 & \\
\hline & Avant la saison pluvieuse & 50 & 25,13 & 11 & 14,86 & \\
& Après la saison pluvieuse & 11 & 5,53 & 4 & 5,41 & \\
& Avant et après la saison pluvieuse & 20 & 10,05 & 8 & 10,81 & \\
Période de & Campagne de vaccination de l'Etat & 91 & 45,73 & 48 & 64,86 & \multirow{2}{*}{ Ns } \\
vaccination & Chaque trimestre & 4 & 2,01 & - & - & \\
& En fonction de l'état des animaux & 21 & 10,55 & 3 & 4,05 & \\
& Après l'achat & 2 & 1,01 & - & - & \\
& Total & 199 & 100 & 74 & 100 & \\
\hline
\end{tabular}

Ns $=$ non significatif $; *=\mathrm{p}<0,05 ; \mathrm{N}=$ effectif $; \%=$ pourcentage. 
Tableau 13 : Pratique du déparasitage des animaux.

\begin{tabular}{llccccl}
\hline \multirow{2}{*}{ Variables } & \multirow{2}{*}{ Modalité } & \multicolumn{2}{c}{ Urbain } & \multicolumn{2}{c}{ Périurbain } & \multirow{2}{*}{ P } \\
\cline { 2 - 5 } Déparasitage & Oui & $\mathbf{N}$ & $\mathbf{\%}$ & $\mathbf{N}$ & $\mathbf{\%}$ & \\
\hline \multirow{2}{*}{ des animaux } & Non & 199 & 75,67 & 74 & 91,40 & \\
& Total & 64 & 24,33 & 7 & 8,60 & $* * *$ \\
& Avant la saison pluvieuse & 263 & 100 & 81 & 100 & \\
& Après la saison pluvieuse & 11 & 36,70 & 23 & 31,08 \\
& Avant et après la saison pluvieuse & 25 & 12,60 & 10 & 13,51 \\
Période de & Campagne de l'Etat & 26 & 13,10 & 26 & 35,14 & \\
déparasitage & Chaque mois & 1 & 0,50 & - & - & $* * * *$ \\
& Chaque trimestre & 8 & 4,00 & 1 & 1,35 & \\
& Chaque 6 mois & 1 & 0,50 & - & - & \\
& en fonction de l'état des animaux & 50 & 25,10 & 11 & 14,86 & \\
& Apres l'achat & 4 & 2,00 & - & - & \\
& Total & 199 & 100 & 74 & 100 & \\
\hline
\end{tabular}

$* * *=\mathrm{p}<0,001 ; \mathrm{N}=$ effectif $; \%=$ pourcentage

Tableau 14 : Déterminants des marges (FCFA).

\begin{tabular}{lllll}
\hline \multirow{2}{*}{ Rubriques } & \multicolumn{3}{c}{ Urbain } & \multicolumn{2}{c}{ Périurbain } \\
\cline { 2 - 5 } & Moyenne & Ecart type & Moyenne & Ecart type \\
\hline Charges & & & & \\
Main d'œuvre & 6000 & 2494,44 & 20406,25 & 18988,69 \\
Conduite au pâturage en saison sèche & 7500 & 6557,04 & 7105 & 6176,91 \\
Conduite pâturage saison pluvieuse & 9086,96 & 7819,92 & 10505,77 & 9565,46 \\
Achat Concentré & 14979,55 & 8980,15 & 22506,67 & 10458,11 \\
Achat Fourrage & 2678,96 & 3599,89 & 37593,47 & 32036,71 \\
Achat supplément & 2343,87 & 785,17 & 2726,43 & 1732,62 \\
Dépenses sanitaires & 425,63 & 320,98 & 195,54 & 74,25 \\
Moyenne charges & 6145 & 4365,37 & 14434,16 & 11290,39 \\
$\quad$ Produits & & & & \\
Vente lait & 121758,62 & 98839,21 & 46326,92 & 48223,22 \\
Vente animaux & 21544,21 & 19867,72 & 19896,34 & 12139,52 \\
Vente fumier & 8396,67 & 15099,18 & 1000 & - \\
Moyenne produits & 50566,5 & 44602,04 & 22407,75 & 30181,37 \\
Marge brute & $\mathbf{4 4 4 2 1 , 5}$ & $\mathbf{4 0 2 3 6 , 6 6}$ & $\mathbf{7 9 7 3 , 5 9}$ & $\mathbf{1 8 8 9 0 , 9 8}$ \\
\hline
\end{tabular}

\section{DISCUSSION}

L'élevage est pratiqué autour de Niamey majoritairement par les ethnies Peul et Zarma (Belli et al., 2008). Ils se caractérisent par une forte représentation $(45,6 \%)$ des Zarmas en milieu urbain, tandis qu'en périurbain ce sont les éleveurs d'ethnie peul qui dominent $(69,1 \%)$. Ces derniers étaient jadis des transhumants qui, aujourd'hui se sont sédentarisés autour des grandes villes pour répondre à une forte demande en lait (Vias et al., 2003). Quant aux Zarmas, ils sont majoritaires dans la communauté urbaine de Niamey et ils représentent le deuxième groupe d'éleveurs le plus important dans cette localité (Boukary et al., 2007).

S'agissant du troupeau d'élevage, il appartient en grande partie aux chefs de 
famille et aux femmes qui sont principalement des petits commerçants, des fonctionnaires et des ménagères (Vias et al., 2003 ; Mani et al., 2013 ; Abdoul, 2014). Ceci pourrait s'expliquer par le fait que ces catégories d'acteurs ont les moyens de se procurer des animaux, parce que possédant des revenus ou par souci de valoriser les résidus de cuisine et de cultures. Cet état de fait peut se justifier également par les raisons les plus importantes de l'élevage qui sont la passion et la tradition. Par conséquent, pour l'éleveur, l'élevage est plus une activité traditionnellement familiale qu'une activité économique (Vias et al., 2003 ; Belli et al., 2008), sans aucune définition d'un objectif de production (Siddo, 2017) .

A travers cette étude, il a été remarqué une prédominance de l'élevage des ovins dans la communauté urbaine de Niamey. Cette situation reflète la réalité du pays où les petits ruminants sont numériquement plus importants que les gros ruminants (Niger, 2007 ; Mani et al., 2013). Les bovins sont généralement élevés en zone périurbaine, le plus souvent en association avec les ovins, principalement en milieu périurbain $(32,10 \%)$. L'association gros ruminants-petits ruminants permet une bonne gestion de l'alimentation d'une part, et d'autre part, l'élevage des petits ruminants est moins pénible et moins exigeant que celui des gros ruminants. Les petits ruminants occupent moins d'espace et ils ont une multiplication et une croissance plus rapides. Ils peuvent être écoulés très facilement du fait de leurs prix relativement bas. En association, l'élevage des petits ruminants est destiné à assurer les dépenses courantes ou saisonnières et celui des gros ruminants à la sécurisation financière et alimentaire des ménages (Madani et al., 2001 ; Corniaux et al., 2009; Alexis et al., 2014). Selon Alexis et al. (2015), cette association permet une répartition des risques épidémiologiques, de l'accès aux ressources et de la sensibilité aux aléas climatiques entre les espèces. De plus, la complémentarité alimentaire des gros et petits ruminants, permet d'accroître la charge à l'hectare et augmenter la productivité des animaux (Alexis et al., 2014).

Par ailleurs, il a été observé une plus grande possession des animaux femelles, avec une prédominance des petits ruminants. Ce même constat a été fait au Niger par Rhissa en 2010. Selon Samuel et Salako (2008), dans une étude conduite au Nigeria, il a été démontré que les femelles sont gardées plus longtemps que les mâles dans un souci de minimiser le coût de l'alimentation et d'améliorer la productivité des animaux.

S'agissant des bovins, il a été observé un nombre de femelle par éleveurs plus important en milieu périurbain qu'en milieu urbain. D'après Bonfoh et al. (2007), Belli et al. (2008), les zones périurbaines représentent le milieu de prédilection de l'élevage bovin laitier. Le peu de mâles qu'on rencontre sont destinés le plus souvent à l'embouche et à la traction.

Dans la communauté urbaine de Niamey, les animaux sont élevés ensemble sans allotement, en grande partie dans des habitats couverts en matériaux provisoires $(57,03 \%$ en milieu urbain et $25,93 \%$ en milieu périurbain) ou à l'air libre sans habitat $(18,63 \%$ en milieu urbain et $46,91 \%$ en milieu périurbain). En milieu urbain, $49,05 \%$ des bovins sont en stabulation entravée contre $49,38 \%$ en milieu périurbain ou à la fois libre et entravée (27,38\% en milieu urbain et $27,16 \%$ en milieu périurbain). Ceci entraîne une pratique d'élevage nécessitant l'utilisation d'une main d'œuvre et un apport d'une grande partie de l'alimentation à l'auge.

Ainsi, 47,91\% des enquêtés de la zone urbaine de Niamey utilise une main d'œuvre dans la conduite de l'élevage, contre 38,27\% en zone périurbaine. En effet, $94,44 \%$ des enquêtés en milieu urbain et $48,39 \%$ en milieu périurbain utilisent cette main d'œuvre pendant toutes l'année sans distinction de saison. Néanmoins, en milieu périurbain, on observe une importante proportion d'utilisateurs de cette main d'œuvre (35,48\%) pendant la saison sèche. Cette dernière est la période pendant laquelle les animaux sont soit en divagation, soit conduit au pâturage. Cependant, cette main d'œuvre est 
principalement familiale avec $86,51 \%$ des cas en milieu urbain et $58,06 \%$ en milieu périurbain. Ainsi, le suivi des animaux repose essentiellement sur les femmes et les enfants de la famille et quelques rares fois sur un berger.

D'un autre côté, l'élevage autour de Niamey a un caractère semi intensif où l'alimentation est apportée aux animaux en grande partie à l'auge. Il ressort de cette étude que les pratiques alimentaires sont fonctions des saisons. La disponibilité de l'herbe fraîche pendant la saison des pluies limite toute utilisation des autres fourrages. En milieu urbain, en saison sèche comme en saison pluvieuse, plus de 68\% des éleveurs alimentent leurs animaux à l'auge. Cette pratique est due en grande partie au manque d'espace de pâture. En milieu périurbain, il ya une certaine variabilité de la conduite alimentaire en fonction des saisons. La divagation et conduite au parcourt nature sont plus pratiquées en saison sèche. Les animaux valorisent les résidus de culture laissés dans les champs après les récoltes. Ce constat rejoint celui de Abdou et al., (2017), à travers une étude conduite à Maradi sur la chèvre rousse. En saison des pluies, c'est surtout l'alimentation à l'auge et la conduite au parcourt naturel qui prédominent. Pour éviter les dégâts, les animaux sont confinés dans les maisons ou conduit au pâturage sous la supervision d'un berger. De retour le soir, il s'en suit une complémentation surtout pour le troupeau laitier (Chaibou et al., 2011). Une étude au Burkina Faso a montré que la complémentation des femelles est quasipermanente pendant l'année. Cette pratique permet d'augmenter la production laitière qui assure une génération de revenus pour l'éleveur. Elle permet également d'assurer la productivité animale et la réduction des risques de mortalité des jeunes animaux et des femelles fatiguées (Zoundi et al., 2003). Ainsi, le concentré le plus utilisé en alimentation animale dans la communauté urbaine de Niamey se trouve être le son de céréale. Il est majoritairement distribué aux animaux deux fois par jour $(42,6 \%$ en milieu urbain et $65,4 \%$ en milieu périurbain) durant toute l'année sans distinction de période $(92,4 \%$ en milieu urbain et $76,5 \%$ en milieu périurbain). L'importance accordée au son de céréales par les éleveurs est surtout due à sa disponibilité et son accès facile, parce qu'il est produit dans presque toutes les familles nigériennes, mais aussi par sa haute valeur nutritive (Rhissa, 2010; Chouidi, 2011).

S'agissant des fourrages, en milieu urbain, ce sont les fanes de niébé et la paille de brousses qui sont abondement utilisées sans préférence de saison. La complémentation avec les fourrages en milieu périurbain de Niamey est relativement faible, de même que la supplémentation dans la communauté urbaine de Niamey.

Concernant la conduite alimentaire, en saison des pluies, les animaux sont plus alimentés à l'auge ou conduits au parcourt naturel par un berger. Notre constat rejoint celle de Moussa et al. (2013) qui soulignent que pendant la saison des pluies, les animaux restent confinés dans les parcs. Elle est contraire à celle d'Ali et al. (2003) qui rapportent qu'en zone périurbaine, les modes de conduite les plus répandus étaient la stabulation combinée au gardiennage en saison sèche; la stabulation permanente en saison des pluies. La conduite des animaux au pâturage par un berger permet d'éviter les dégâts champêtres qui sont parfois des sources de conflits entre agriculteurs et éleveurs (Vias et al., 2003). En saison sèche, on observe une baise de la pratique d'alimentation à l'auge et une augmentation de la divagation des animaux. Il résulte de ce phénomène, une valorisation des résidus de cultures laissés au champ. Ces résultats corroborent ceux fournis par le recensement général de l'agriculture et du cheptel en 2007; mais aussi, par Kassoumna (2009) à travers une étude menée au Tchad sur la mobilité et la sédentarisation.

La vaccination et le déparasitage sont pratiqués par les éleveurs de la communauté urbaine de Niamey. Mais, hormis les périodes de campagne gratuite de vaccination parfois couplées avec un déparasitage, rares sont les éleveurs de la communauté urbaine de Niamey qui vaccinent et déparasitent leurs animaux. 
Sur le plan économique, les résultats de cette étude ont montré que le chiffre d'affaire tiré de l'élevage par les éleveurs de la communauté urbaine de Niamey repose essentiellement sur la vente des animaux sur pieds, celle du lait et du fumier. Ainsi, il en ressort qu'un éleveur urbain gagne mensuellement 6 fois (44421,5 FCFA) plus qu'un éleveur périurbain (7973,59 FCFA). Cet écart est dû en grande partie à l'orientation donnée à l'élevage et aux charges y afférentes. En effet, en milieu urbain, l'élevage est plus une activité économique que sociale. Ce qui fait qu'il nécessite plus d'investissement et de suivi. C'est un élevage de type semi intensif avec une forte utilisation des concentrés, d'infrastructures et de mains d'œuvre salariale (Sanon et al., 2014). La vente des animaux est très fréquente pour un souci de gestion des espaces et du coût de l'alimentation. En milieu périurbain, c'est plutôt un élevage laitier, d'héritage, de prestige et d'épargne. Ce qui implique une faible sortie des animaux. Ces résultats rejoignent ceux de Karimou (2015), qui affirmait que 95\% des recettes liées au secteur de l'élevage proviennent de la vente d'animaux sur pied. Les recettes provenant de la vente des produits d'origines animales demeurent très faibles et proviennent essentiellement de la vente du lait.

\section{Conclusion}

Cette étude a permis de mieux distinguer l'importance de l'élevage dans la communauté urbaine de Niamey. Il en ressort que la passion et l'héritage sont les principales raisons de la pratique de l'élevage dans cette localité, avec des animaux le plus souvent élevés en agrégation mixte dans des habitats couverts en matériaux provisoires ou à l'air libre sans habitat. Le chiffre d'affaire repose essentiellement sur la vente des animaux sur pieds, celle du lait et du fumier.

En somme, les pratiques d'élevage dans la communauté urbaine de Niamey restent encore traditionnelles avec des orientations de production pas très bien définit. Il est donc impératif de sensibiliser les éleveurs sur les objectifs à assigner à leur élevage afin de mieux organiser l'agrégation et les pratiques alimentaires. Cela permettra de bien définir les objectifs de production et facilitera l'atteinte de ces objectifs tout en rentabilisant cet élevage.

\section{CONFLIT D'INTERETS}

Les auteurs de ce manuscrit déclarent qu'il n'y a aucun conflit d'intérêts entre eux.

\section{CONTRIBUTIONS DES AUTEURS}

Dans la présente étude, AAML a assuré l'élaboration du protocole de recherche, la collecte, le traitement des données et la rédaction du manuscrit sous l'encadrement et la supervision de MC et ASG; MM et MMG ont contribué à la relecture des différentes versions pour l'amélioration de la qualité scientifique du manuscrit.

\section{REMERCIEMENTS}

Les auteurs remercient le Programme de Productivité Agricole de l'Afrique de l'Ouest (PPAAO-Niger) à travers le Centre National de Spécialisation en Elevage pour avoir financé ce travail.

\section{REFERENCES}

Abdou MM, Issa S, Dan Gomma A. Sawadogo GJ. 2017. Analyse technicoéconomique des Aliments densifies sur les performances de croissances des boucs roux de Maradi au Niger. Int. J. Biol. Chem. Sci., 11(1): 280-292. DOI: http://dx.doi.org/10.4314/ijbcs.v11i1.22.

Abdoul ML. 2014. Aspects socioéconomiques de l'utilisation des résidus de cultures et des Sous-produits agroindustriels dans l'alimentation des ruminants domestiques à Niamey (Niger). Mémoire de Master Productions Animales et Développement Durable, EISMV / Dakar, Dakar, p. 43.

Alexis S, Angeon V, Arquet R, Boval M. 2015. Les systèmes mixtes d'élevage de petits ruminants et de bovins : Une alternative pour améliorer les performances animales au pâturage. Innovations Agronomiques, 43: 19-28. 
Alexis S, Periacarpin F, Jackson F, Boval M. 2014. Mixed grazing systems of goats with cattle in tropical conditions: an alternative to improve animal production at pasture. Animal., 8(8): 1282-1289. DOI: $10.1017 / \mathrm{S} 1751731114001542$.

Ali L, Van den Bossche P, Thys E. 2003. Enjeux et contraintes de l'élevage urbain et périurbain des petitsruminants à Maradi au Niger : quel avenir?. Revue Élev. Méd. Vét. Pays. Trop., 56(1-2): 7382.

Beidari S, 1999. Bilan et perspectives de la production laitière en zone périurbaine au Niger: cas de la Commune Urbaine de Niamey. IPR/IFRA, Bamako, 120 p.

Belli P, Turini J, Harouna A, Garba IA, Pistocchini E, Zecchini M. 2008. Critères de sélection des bovins laitiers par les éleveurs autour de Niamey au Niger. Revue. Élev. Méd. Vét. Pays. Trop., 61(1): 51-56.

Bonfoh B, Fokou G, Ould Taleb M, Fané A, Woirin D, Laimaibao N, Zinsstag J. 2007. Dynamiques des systèmes de production laitière, risques et transformations socio-économiques au Mali. Revue. Élev. Méd. Vét. Pays. Trop., 60(1-4) : 67-76.

Boukary AR, Chaïbou M, Marichatou H, Vias G. 2007. Caractérisation des systèmes de production laitière et analyse des stratégies de valorisation du lait en milieu rural et périurbain au Niger : cas de la communautéurbaine de Niamey et de la commune rurale de Filingué. Revue. Élev. Méd. Vét. Pays. Trop., 60(1-4) : 113-120.

Chaibou M, Illia AS, Marichatou H, 2011. Pratiques de gestion et performances de production dans les élevages bovins laitiers urbains et périurbains de Niamey. Revue des Bio Ressources., 1(2): 1-12.

Chouidi B. 2011. Impact de la crise des matières premières agricoles sur la commercialisation des intrants d'aliment du bétail dans la communauté urbaine de Niamey (NIGER). Mémoire de master productions Animales et Développement Durable, EISMV/Dakar, Dakar, p. 44.
Corniaux C, Alary V, Cloarec M. 2009. Poids de l'élevage dans l'économie monétaire des ménages et vulnérabilité des agroéleveurs en zone Office du Niger (Mali). Renc. Rech. Rum., 16: 341-344.

Faye B. 2009. Pauvreté et solidarité chez les peuples pastoraux. In L'Elevage, Richesse des Pauvres, Duteurtre G, Faye B (Eds). Editions Quae : France ; 77-87. DOI: 10.3917/quae.duteu.2009.01.0077

Habou A Z, Boubacar MK, Adam T. 2016. Les systèmes de productions agricoles du Niger face au changement climatique : défis et perspectives. Int. J. Biol. Chem. Sci., 10(3): 1262-1272. DOI : http://dx.doi.org/10.4314/ijbcs.v10i3.28.

Karimou BH. 2015. Caractérisation phénotypique et zootechnique de la chèvre rousse de Maradi. Thèse de Doctorat en Productions Animales, Université Abdou Moumouni de Niamey, Niamey, p. 138.

Kossoumna LN. 2009. De la mobilité à la sédentarisation: gestion des ressources naturelles et des territoires par les éleveurs m'bororo au nord du Cameroun. Thèse de doctorat en géographie, Université Paul Valéry/ Montpellier III, Montpellier, p. 284.

Lawal MA, Chaibou M, Garba MM, Mani M, Gouro AS. 2017. Gestion et utilisation des résidus de cultures pour l'alimentation animale en milieu urbain et périurbain: cas de la communauté urbaine de Niamey. Journal of Applied Biosciences, 115: 11423-11433. DOI: https://dx.doi.org/10.4314/jab/v115i1.2.

Madani T. Hubert B, Lasseur J, Guérin G. 2001. Association des bovins, des ovins et des caprins dans les élevages de la Suberaie algérienne. Cahiers Agricultures, 10: 9-18.

Mani M, Marichatou H, Moumouni I, Sow A, Chaibou I, Chaibou M, Sawadogo GJ. 2013. Les pratiques d'élevage caprin au Niger. RASPA., 11(2) : 107-116.

Moussa GM, Marichatou H, Issa M, Abdoul Aziz ML, Hanzen C. 2013. Tractus génital des vaches zébus (Bosindicus) au 
Niger. Revue. Élev. Méd. Vét. Pays. Trop., 66(4) : 137-142.

Niger. 2007. Recensement Général de l'Agriculture et du Cheptel (RGAC). Résultats définitifs Volume II (Volet cheptel) : Niamey.

Niger. 2013a. L'élevage et les conditions de vie des ménages au Niger. Une analyse descriptive de l'enquête sur les conditions de vie des ménages et l'agriculture. Ministère de l'élevage : Niamey.

Niger. 2013b. Stratégie de Développement Durable de l'Elevage, 2013-2035. SDDEL, Niamey.

Niger. 2015a. Annuaire statistique régional 2010 - 2014. Institut national de la statistique, Niamey.

Niger. 2015b. Synthèse des résultats de la campagne pastorale 2015-2016 : Version provisoire. Ministère de l'élevage : Niamey.

Niger. 2016. Recensement général de la population et de l'habitat 2012. Institut national de la statistique : Niamey.

Rhissa Z. 2010. Revue du secteur de l'élevage au Niger. Rapport provisoire. FAO/SFW : Niamey.

Samuel FOK, Salako AE. 2008. Body measurement characteristics of the West African Dwarf (WAD) Goat in deciduous forest zone of Southwestern Nigeria. African Journal of Biotechnology, 7(14): 2521-2526. http://www.academicjournals.org/AJB.

Sanon HO, Drabo A, Sangaré M, Kiendrebeogo T, Gomgnibou A. 2014. Caractérisation des pratiques d'embouche bovine dans l'Ouest du
Burkina Faso. Int. J. Biol. Chem. Sci., 8(2): $\quad 536-550 . \quad$ DOI: http://dx.doi.org/10.4314/ijbcs.v8i2.13.

Siddo S. 2017. Evaluation socio-économique du potentiel de diffusion du zébu Azawak sélectionné au Niger. Thèse de Doctorant en Sciences Vétérinaires, Université de Liège, Liège, p. 145.

Steinfeld H, Mooney HA, Scheider F, Neville LE. 2010. Livestock in a Changing Landscape: Drivers, Consequences and Responses (vol 1). Island Press: Washington.

Vias FSG, Bonfoh B, Naferi A, Faye B. 2003. Les élevages laitiers bovins autour de la Communauté Urbaine de Niamey: Caractéristiques, production, commercialisation et qualité du lait. Etud. Et Rech. Saheliennes, 8-9: 159165.

Waters-Bayers A. 2000. Living with livestock in town. Urban. Agric. Mag., 1: 16-20.

Zoffoun AG, Aboh AB, Adjolohoun S, Houinato M, Sinsin B. 2013. Effet de l'âge et de l'intensité de pâture sur le développement des touffes et la production de biomasse de Panicum maximum var. $\mathrm{C} 1$ dans les pâturages artificiels en zone soudanienne et subéquatoriale. Int. J. Biol. Chem. Sci., 7(3): $\quad 1168-1179 . \quad$ DOI: http://dx.doi.org/10.4314/ijbcs.v7i3.23.

Zoundi JS, Sawadogo L, Nianogo AJ. 2003. Pratiques et stratégies paysannes en matière de complémentation des ruminants au sein des systèmes d'exploitation mixte agriculture-élevage du plateau central et du Nord du Burkina Faso. Tropicultura, 21(3): 122-128. 